\title{
HOMONUCLEAR SEPARATED LOCAL-FIELD SPECTROSCOPY BY MAGNETIZATION-EXCHANGE NMR
}

\author{
D.E. DeMCo*
}

Department of Physics, Technical University, 3400 Cluj-Napoca, Romania

\author{
X. Filip, C. Filip
}

Institute for Isotopic and Molecular Technology, 3400 Cluj-Napoca, Romania

D. Chira and O. Cozar

Faculty of Physics, University Babes-Bolyai, 3400 Cluj-Napoca, Romania

(Received July 7, 1997)

The possibility to perform homonuclear separated-local field NMR spectroscopy by magnetization-exchange is presented. Solid-state one-dimensional proton magnetization-exchange NMR is used to investigate intergroup residual dipolar couplings in cis-1,4-polybutadiene elastomer. A three-spin model is employed, in which the $\mathrm{CH}$ - and $\mathrm{CH}_{2}$-protons are considered to be coupled by residual dipolar interactions. The magnetization-exchange process between the $\mathrm{CH}$ - and the $\mathrm{CH}_{2}$-group in the regime of short mixing time provides valuable insight regarding molecular order. The spin-system response reflects well-localized dipolar interactions. The residual intergroup dipolar couplings are measured along the average polymer-chain direction for different temperatures. The dynamic order parameter along the chains is also evaluated.

PACS numbers: $33.25 .+k, 61.41 .+\mathrm{e}$

\section{Introduction}

Separated local-field spectroscopy is a general approach for the resolution and measurements of spectral parameters associated with the homonuclear and heteronuclear dipolar interactions between abundant spins (e.g. ${ }^{1} \mathrm{H},{ }^{19} \mathrm{~F}$ or ${ }^{31} \mathrm{P}$ ) and between an abundant $I$ spin(s) and a specific dilute $S$ spin $\left(\right.$ e.g. ${ }^{13} \mathrm{C}$ or ${ }^{15} \mathrm{~N}$ ),

*Corresponding author. 
respectively, as a result of the spectroscopic resolution accorded especially by the dilute spin chemical shift frequencies.

In solids and liquid crystalline phase, two-dimensional (2D) spectroscopy may be used to separate and correlate the anistropic chemical shifts and dipolar couplings in analogy to 2D separation in liquids $[1,2]$. The resulting spectra with dipolar multiplets separated by chemical shifts are known as separated local-field (SLF) spectra and were introduced by Waugh [3]. Although separated local-field spectroscopy is closely associated with the original pulse sequence [3], many variations have been developed and applied to single crystals, oriented materials, and powders in the presence and absence of magic-angle sample spinning (MAS) [4-21].

Multiple-quantum NMR spectroscopy is a valuable tool for the identification and characterization of spin system. This method was used for measuring heteronuclear [22] and homonuclear dipolar couplings, i.e., to perform SLF, for simple molecules oriented in liquid crystals [23-25]. In recent years there has been a sustained effort to obtain homonuclear and heteronuclear high-resolution $\mathrm{MQ}$ spectra in solids with dipolar-coupled spins [26-29], using fast MAS for increased resolution. The main aim of such experiments are structural and dynamic studies of solids by probing dipolar connectivities as well as local-field spectroscopy.

The aim of this paper is to discuss the possibility to perform homonuclear SLF by magnetization-exchange experiments in rigid solids and quasi-solids. As an example for the last case we consider a particular elastomer. Homonuclear ${ }^{1} \mathrm{H}-{ }^{1} \mathrm{H}$ dipolar couplings are generally so strong in solids that they present major difficulties for the resolution of resonances and the selective measurements of spectral parameters. However, homonuclear proton magnetization exchange has been observed in elastomers [30-33]. Elastomers represent an important class of materials for which magnetization exchange using $2 \mathrm{D}$ spectroscopy is advantageous. A combination of MAS and 2D nuclear Overhauser-effect spectroscopy (NOESY) [30] (and references therein) has been used for investigation of intermolecular interactions which are responsible for the miscibility of polymer blends, for instance 1,2-polybutadiene/polyisoprene. Also, the effect of chemical cross-linking in technical elastomers such as poly(styrene-co-butadiene) (SBR), has been the subject of first 2D investigations [31-33]. The purpose of these studies was to relate molecular structure and dynamics to macroscopic material properties.

From the viewpoint of NMR, cross-linked elastomers exhibit both, solid-like and liquid-like features. Whereas the segmental motions give rise to the liquid-like behavior, the presence of permanent cross-links leads to residual dipolar couplings, that are responsible for the solid-like properties. Recently, the heterogeneous nature of the residual dipolar couplings resulting from the constrained chain motions in these systems has been elucidated by $2 \mathrm{D}$-exchange NMR $[32,33]$. Moreover, it was shown $[32,33]$ for a series of vulcanized SBR elastomers that the concept of residual dipolar couplings can be used to characterize quantitatively the influence of the cross-link density on several NMR parameters. At the core of this model is the assumption of localized homonuclear and heteronuclear dipolar interactions for the short time scale of the NMR experiment.

In Sec. 2 we shall discuss the features of three-spin model valid for cis-1,4-polybutadiene. This model is used for discussing in Sec. 4 the chemical 
shift filters as well as magnetization-exchange dynamics. The measured quantity is the intergroup residual dipolar coupling which is presented in Sec. 3. The one-dimensional magnetization-exchange experiment is described in Sec. 5 and the measurement of residual dipolar coupling between $\mathrm{CH}$ and $\mathrm{CH}_{2}$ functional groups in cis-1,4-polybutadiene is presented in Sec. 6.

\section{Spin-system model}

The model which will be considered in the following to describe the proton magnetization-exchange in cis-1,4-polybutadiene is based on the following assumptions and properties of the polymer chain:

(i) The majority of functional groups $\left(a \equiv \mathrm{CH}_{2}, b \equiv \mathrm{CH}\right)$, in cis-1,4-polybutadiene are arranged in a pair sequence $a a b b a a .$. This pair sequence is present in trans- and cis-1,4-butadiene units as well as in 1,2-butadiene units in the case of head-to-head- or tail-to-tail polymerization [34]. The alternating sequence $a b a b . .$. is less frequent. The relevant spin system is depicted in Fig. 1.

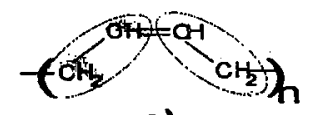

a)

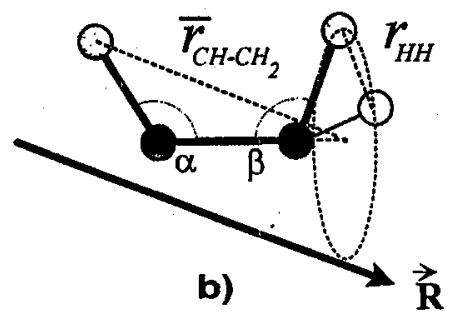

Fig. 1. (a) The monomer units of cis-1,4-butadiene. The alternating sequence of $\mathrm{CH}-\mathrm{CH}_{2}$ groups, for which the magnetization-exchange process is treated, is shown by the shaded area. (b) The geometry of the $\mathrm{CH}-\mathrm{CH}_{2}$ spin system. The shaded circles represent protons and the black circles carbon atoms. The conformational jumps of the methylene protons lead to an average $\mathrm{CH}-\mathrm{CH}_{2}$ proton distance $\bar{r}_{\mathrm{CH}-\mathrm{CH}_{2}}$. The angles $\alpha$ and $\beta$ are $120^{\circ}$ and the tetrahedral angle, respectively.

(ii) Topological constraints (as well as chemical cross-links) lead to restrictions of the chain motions and hence to a non-zero average of the dipolar interactions between the protons of the $\mathrm{CH}_{2-}$ and the $\mathrm{CH}$-group along the chain. This is predominantly the case within each repeat unit and perhaps also between neighboring units. Depending on the degree of motional averaging, these residual dipolar interactions may be scaled to quite small values. They reflect single-chain properties because the fast molecular motions of the polymer chains effectively average the interchain dipolar interactions. The validity of these assumptions was proved by investigations of the ${ }^{1} \mathrm{H}$-transverse relaxation during a solid-echo pulse train and by a 2D magnetization-exchange experiment performed with a short 
mixing time [33]. The fast fluctuating part of the dipolar couplings also leads to magnetization-exchange. However, it will be shown that this exchange occurs at much lower rates. Thus, in a substantial temperature range above the glass transition, the evolution of the coherences in the network is determined mainly by residual dipolar interactions. We therefore expect that for short and intermediate mixing times the solid-like (coherent, reversible, or deterministic) behavior will be dominant. However, for longer mixing times, incoherent (irreversible or stochastic) cross-relaxation processes [1] will also take place due to slow chain dynamics.

(iii) Fast anisotropic molecular motions lead to partially narrowed static proton spectra for polybutadiene. At room temperature, two lines are resolved in the static proton spectrum presented in Fig. 2. One line corresponds to the $\mathrm{CH}$-groups and the other to the $\mathrm{CH}_{2}$-groups. They are separated by $3.35 \mathrm{ppm}$, that is by about $1 \mathrm{kHz}$ at the Larmor frequency of $300 \mathrm{MHz}$.

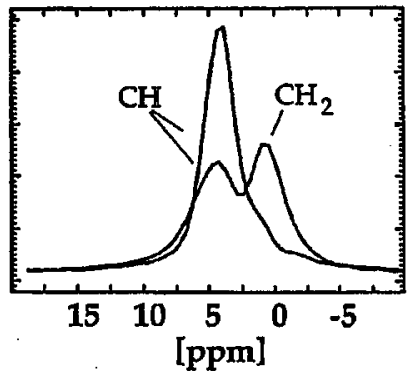

Fig. 2. Proton spectra recorded in a $1 \mathrm{D}$ magnetization-exchange experiment using the CH-chemical-shift filter $\left(t_{1}=260 \mathrm{~ms}\right)$. For the short mixing time of $t_{m}=$ $500 \mu \mathrm{s}$, the spectrum shows predominantly the $\mathrm{CH}$-line. For $t_{m}=15 \mathrm{~ms}$, the magnetization-exchange process leads to the reappearance of the $\mathrm{CH}_{2}$ line at the expense of the signal intensity of the $\mathrm{CH}$ line.

(iv) For short mixing times, the measurable exchange of longitudinal proton magnetization predominantly takes place between the protons belonging to nearest-neighbor $\mathrm{CH}$ - and $\mathrm{CH}_{2}$-groups. The spin system that has to be considered for describing this process thus is a three-spin system in which the two strongly coupled protons of the $\mathrm{CH}_{2}$-group interact with the remote proton of the $\mathrm{CH}$-group. The dipolar couplings between two neighboring $\mathrm{CH}$ groups or two neighboring $\mathrm{CH}_{2}$ groups, respectively, are not relevant with respect to our measurements of the magnetization-exchange dynamics at short mixing times, since even after application of the chemical-shift filter [2] they are in thermodynamical equilibrium among themselves. Moreover, the size of the corresponding dipolar reservoirs is quite small compared with the Zeeman reservoirs. That is, the small value of the residual intrachain couplings allows one to consider the exchange within a fairly isolated spin system for short mixing times. Together with the arguments presented in (i), this further supports the validity of the three-spin model. At longer mixing times, however, a much larger spin system along the polymer chain must be considered [33]. 
Based on the above, we will discuss in the following quantitatively the magnetization-exchange dynamics in the limit of short mixing times. As outlined above, it is sufficient to treat the simple model system of three proton spins coupled by static residual dipolar interactions.

\section{Residual dipolar couplings}

It is natural to treat the residual couplings resulting from entanglements or physical cross-link (as well as chemical cross-link) by statistical chain models. Corresponding calculations have been carried out by several authors ([32] and references therein). Using the simplified scale-invariant model discussed in Ref. [32], the average residual dipolar couplings within a chain of $N$ statistical segments can be written as

$$
\bar{D}^{\mathbb{g}} \equiv D_{i j}^{\mathrm{g}}\left(\frac{4 \pi}{5}\right)^{\frac{1}{2}} \frac{k}{N^{2} a^{2}} R^{2} Y_{2,0}(\Omega)
$$

The dipolar coupling constant of the two protons $(i j)$ that belong to the same ( $\mathrm{g}=$ $\left.\mathrm{CH}_{2}\right)$ or to different functional groups $\left(\mathrm{g}=\mathrm{CH}-\mathrm{CH}_{2}\right)$ is $D_{i j}^{\mathrm{g}}=\left(\frac{\mu_{0}}{4 \pi}\right) \frac{\gamma^{2} \hbar}{r_{i j}^{3}}$, where the symbols are defined following Ref. [2]. The parameter $N$ describes the influence of the long-range structure, i.e., the physical cross-link density. The length of a statistical segment is denoted by $a$. The geometrical factor $k$ depends on the model that is adopted for describing the chain statistics. Therefore, the parameter $N$ should not be identified as the actual number of repeat units between the physical cross-links, but strictly speaking is only related to it. In particular, $N$ may be strongly biased by physical cross-links due to local order $[32,35]$, where the average chain length between them is significantly shorter than the length between the chemical cross-links.

In order to evaluate the statistical average of the squared residual dipolar coupling (vide infra), we suppose that the end-to-end vector components $(x, y, z)$ obey ideal Gaussian statistics with a normalized distribution given by Ref. [36]

$$
P(\boldsymbol{R}) \mathrm{d} \boldsymbol{R}=\left(\frac{3}{2 \pi N a^{2}}\right)^{\frac{3}{2}} \exp \left(-\frac{3}{2} \frac{\boldsymbol{R}^{2}}{N a^{2}}\right) \mathrm{d} x \mathrm{~d} y \mathrm{~d} z .
$$

The orientational average of the disordered chain distribution and the average over the end-to-end vectors lead to a general formula

$$
\left\langle\left(\bar{D}^{\mathrm{g}}\right)^{2}\right\rangle=\frac{1}{20 N_{\mathrm{e}}^{2}}\left(D_{i j}^{\mathrm{g}}\right)^{2}
$$

in which $N_{\mathrm{e}}$ is introduced to include the factor $k$, i.e., $N_{\mathrm{e}}=N / k$.

At this point we introduce an effective residual dipolar coupling defined by $D_{\mathrm{eff}}^{\mathrm{g}} \equiv\left\langle\left(\bar{D}^{\mathrm{g}}\right)^{2}\right\rangle^{\frac{1}{2}}$ that scales with the adjustable parameter $N_{\mathrm{e}}$ as $D_{\mathrm{eff}}^{\mathrm{g}} \propto N_{\mathrm{e}}^{-1}$. As mentioned above, relating $N_{\mathrm{e}}$ to the actual length quantitatively would require a detailed investigation of local motions and conformations of the chains which is beyond the scope of this work. Moreover, in the above average, the polydispersity has also to be taken into account [37]. 


\section{Magnetization exchange dynamics}

\subsection{Spin Hamiltonian}

A spin Hamiltonian appropriate for the three-spin model discussed in Sec. 2 is considered that describes the residual proton interactions under the conditions of motional preaveraging by fast molecular motions. In the rotating-frame representation $[1,2]$, the Hamiltonian for a static sample is given by

$$
H=H_{\mathrm{Z}}^{\mathrm{CH}}+H_{\mathrm{Z}}^{\mathrm{CH}_{2}}+\bar{H}_{\mathrm{d}}^{\mathrm{CH}_{2}}+\bar{H}_{\mathrm{d}}^{\mathrm{CH}-\mathrm{CH}_{2}} \text {. }
$$

The chemical-shift anisotropy and heteronuclear couplings are neglected in Eq. (4). Thus, the Zeeman Hamiltonians are

$$
H_{\mathrm{Z}}^{\mathrm{CH}}=-\Delta \omega_{\mathrm{CH}} I_{\mathrm{Z}}, \quad H_{\mathrm{Z}}^{\mathrm{CH}_{2}}=-\Delta \omega_{\mathrm{CH}_{2}} F_{\mathrm{Z}},
$$

where the off-resonance prefactors are $\Delta \omega_{i}=\left(\omega_{0}+\delta_{i}\right)-\omega$, for $i=\mathrm{CH}$ and $\mathrm{CH}_{2}$, where the symbols have the significance given in Refs. $[1,2]$. The spin operator for the proton of the CH-group is denoted by $I$ and the spin- $1 / 2$ pair represented by the methylene protons can be replaced by a quasi-particle with spin $F=1$ and a total spin operator $\boldsymbol{F}=\boldsymbol{I}_{1}+\boldsymbol{I}_{2}$. The intra- and intergroup residual dipolar Hamiltonians may be expressed in secular approximation as [33]

$$
\begin{aligned}
& \bar{H}_{\mathrm{d}}^{\mathrm{CH}_{2}}=\bar{d}^{\mathrm{CH}_{2}} Y_{2,0}(\Omega) T_{2,0}^{\mathrm{CH}_{2}}, \\
& \bar{H}_{\mathrm{d}}^{\mathrm{CH}-\mathrm{CH}_{2}} \cong \bar{d}^{\mathrm{CH}-\mathrm{CH}_{2}} Y_{2,0}(\Omega) T_{2,0}^{\mathrm{CH}-\mathrm{CH}_{2}},
\end{aligned}
$$

where $\bar{d}^{j}\left(j=\mathrm{CH}_{2}, \mathrm{CH}-\mathrm{CH}_{2}\right)$ denote the strengths of the residual dipolar couplings. Restrictions to the chain motions leading to residual dipolar coupling result from local chain order, for example due to the presence of extended confirmations or stiffening elements on a short length scale, and entanglements as well as permanent chemical cross-links on a larger scale. The former are also present in a polymer melt, the last is characteristic of elastomers. The spherical harmonics $Y_{2,0}(\Omega)$ depend on the azimuthal angle between the local chain vector $\boldsymbol{R}$ and the direction of the magnetic field $\boldsymbol{B}_{0} . T_{2.0}^{j}$ are irreducible tensor-operators [2]. The distance between the functional groups is fluctuating due to local conformational jumps. Therefore, the $\mathrm{CH}-\mathrm{CH}_{2}$ dipolar coupling of the protons is approximated by the dipolar coupling between the $\mathrm{CH}$-proton and an effective spin-1 nucleus located at an average distance $\bar{r}_{\mathrm{CH}-\mathrm{CH}_{2}}$ (cf. Fig. 1).

The spin Hamiltonian given by Eq. (4) describes only the average (considered to be static) of the dipolar interactions. These residual couplings directly manifest themselves in spinning side bands as shown in Refs. [33,38]. The fluctuating part of the dipolar Hamiltonian is responsible for cross-relaxation by zero-, single- and double-quantum transitions [1]. 


\subsection{Chemical-shift filters}

For the 1D magnetization-exchange experiment, the modified three-pulse sequence of Fig. 3 is applied. This sequence usually is employed to record 2D-exchange spectra $[1,2]$. As described below, it can also be used to record individual slices which is advantageous for the study of the selected exchange signal as a function of the mixing time $t_{m}$. In the $1 \mathrm{D}$ version of the magnetization-exchange experiment, the separation of the NMR lines enables one to apply a chemical-shift filter [2] in order to select the longitudinal magnetization of either the $\mathrm{CH}$ - or the $\mathrm{CH}_{2}$-group. The sequence starts with an evolution period that acts as a chemical-shift filter by an appropriate choice of the rf-irradiation frequency and the duration $t_{1}$. The remaining transverse magnetization after the second $90^{\circ}$ pulse and the excited multiple-quantum coherences are removed from the acquired signal by phase cycling and the application of a short gradient pulse of duration $t_{\mathrm{d}}$ where $t_{\mathrm{d}} \leq t_{m}$ (cf. Fig. 3). As an example, the selection of the $\mathrm{CH}$ signal is shown in Fig. 2. After a mixing time of $t_{m}=15$ ms magnetization exchange is complete and the $\mathrm{CH}_{2}$ signal is fully recovered at the expense of the $\mathrm{CH}$ intensity.

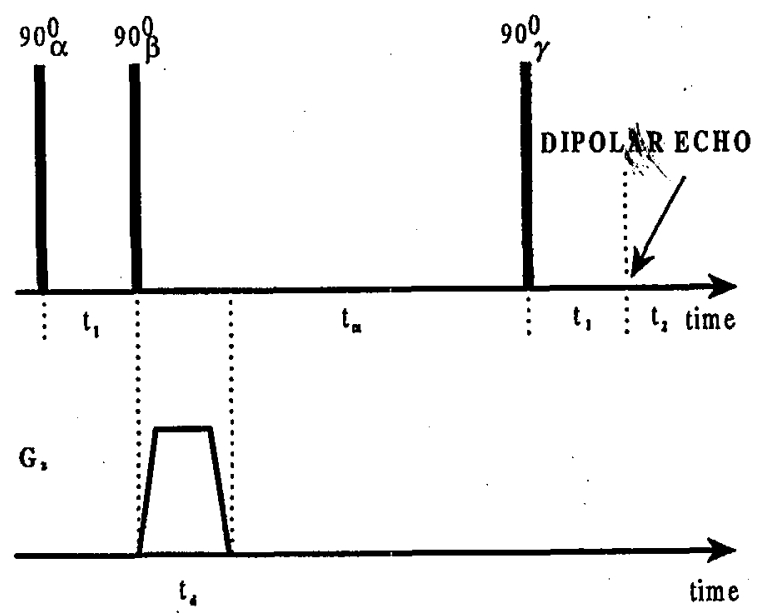

Fig. 3. Basic scheme for magnetization-exchange spectroscopy. The delays $t_{1}, t_{2}$ and $t_{m}$ correspond to the evolution-, detection- and mixing periods. In the $1 \mathrm{D}$ experiments with short mixing times, a pulsed magnetic field gradient is applied during the dephasing period $t_{\mathrm{d}}$.

The CH-group is selected by the conditions $\Delta \omega_{\mathrm{CH}}=0$ and $\Delta \omega_{\mathrm{CH}_{2}} t_{1}=\frac{\pi}{2}$. The corresponding density operator at the beginning of the mixing period after a very short dephasing period of duration $t_{\mathrm{d}}$ is given by

$$
\rho^{\mathrm{CH}}\left(t_{1}\right)=a I_{\mathrm{Z}},
$$

where $a$ is the prefactor of the density operator of thermal equilibrium in the high-field/high-temperature approximation [1] and the dipolar coupling to the remote $\mathrm{CH}_{2}$ group is neglected. 
Likewise, the $\mathrm{CH}_{2}$-group is selected by the conditions $\Delta \omega_{\mathrm{CH}_{2}}=0$, and $\Delta \omega_{\mathrm{CH}} t_{1}=\frac{\pi}{2}$. In this case, the density operator at the beginning of the mixing period is

$$
\rho^{\mathrm{CH}_{2}}\left(t_{1}\right)=a\left\langle\cos \left(\sqrt{\frac{3}{2}} \bar{D}^{\mathrm{CH}_{2}} t_{1}\right)\right\rangle F_{\mathrm{Z}},
$$

where $\bar{D}^{\mathrm{CH}_{2}} \equiv \bar{d}^{\mathrm{CH}_{2}} Y_{2,0}(\Omega)$ and the symbol $\langle\ldots\rangle$ represents the statistical ensemble average $[27,28,30]$ of the space part of the dipolar coupling in the disordered elastomer.

It is evident from Eq. (8) that the strength of the selected $\mathrm{CH}_{2}$ signal depends on the strength of the residual dipolar coupling of the two protons within the methylene group.

\subsection{Magnetization-exchange observables}

The NMR observables of the 1D magnetization-exchange experiment with chemical-shift filters are

$$
\left\langle\left\langle I_{Z}\left(t_{m}\right)\right\rangle\right\rangle=\left\langle\frac{\operatorname{Tr}\left\{I_{\mathrm{Z}} \rho^{\mathrm{CH}}\left(t_{m}+t_{1}\right)\right\}}{\operatorname{Tr}\left\{I_{\mathrm{Z}} \rho^{\mathrm{CH}}\left(t_{1}\right)\right\}}\right\rangle
$$

and

$$
\left\langle\left\langle F_{\mathrm{Z}}\left(t_{m}\right)\right\rangle\right\rangle=\left\langle\frac{\operatorname{Tr}\left\{F_{\mathrm{Z}} \rho^{\mathrm{CH}_{2}}\left(t_{m}+t_{1}\right)\right\}}{\operatorname{Tr}\left\{F_{\mathrm{Z}} \rho^{\mathrm{CH}_{2}}\left(t_{1}\right)\right\}}\right\rangle .
$$

The invariants of the spin evolution that define the effective thermodynamic reservoirs correspond to the $I$ - and $F$-spir Zeeman reservoir and to the "spin 1" dipolar reservoir. During the magnetization-exchange process, all three reservoirs are coupled by the intergroup dipolar interaction.

In order to proceed further, it is of advantage to introduce a new quantum-mechanical representation defined by the canonic transformation with the total Hamiltonian of the spin system reservoirs

$$
\tilde{\rho}(t)=\exp \left[-\mathrm{i}\left(\boldsymbol{H}_{\mathrm{Z}}^{\mathrm{CH}}+\boldsymbol{H}_{\mathrm{Z}}^{\mathrm{CH}_{2}}+\overline{\boldsymbol{H}}_{\mathrm{d}}^{\mathrm{CH}_{2}}\right) t\right] \rho(t),
$$

where $\boldsymbol{H}$ denotes a Liouville Hamiltonian operator [1]. In this representation, the solution of the Liouville-von Neumann equation is given by

$$
\widetilde{\rho}^{(i)}\left(t_{m}+t_{1}\right)=T \exp \left[-\mathrm{i} \int_{0}^{t_{m}} \tilde{\overline{\boldsymbol{H}}}_{\mathrm{d}}^{\mathrm{CH}-\mathrm{CH}_{2}}(t) \mathrm{d} t\right] \rho^{(i)}\left(t_{1}\right)
$$

with $i=\mathrm{CH}$ or $\mathrm{CH}_{2}$, corresponding to the selected initial conditions, i.e., the different chemical-shift filters. $T$ is the time-ordering Dyson operator and $\widetilde{\widetilde{H}}_{\mathrm{d}}{ }^{\mathrm{CH}} \mathrm{CH}_{2}(t)$ is the intergroup Liouville Hamiltonian of the residual dipolar interaction written in the representation defined by Eq. (11).

In the limit of short mixing times defined by $\left\|\widetilde{\widetilde{\boldsymbol{H}}}^{\mathrm{CH}-\mathrm{CH}_{2}}\right\| t_{m} \ll 1$, where $\|\ldots\|$ is the norm of the Hamiltonian, an expansion of the mixing propagator up to the second power can be written as 


$$
\begin{aligned}
& \widetilde{\rho}^{(i)}\left(t_{m}+t_{1}\right) \\
& \cong\left[1-\mathrm{i} \widetilde{\overline{\boldsymbol{H}}}_{\mathrm{d}}^{\mathrm{CH}-\mathrm{CH}_{2}}\left(t_{m} ; 0\right)-\frac{1}{2} \widetilde{\boldsymbol{H}}_{\mathrm{d}}^{\mathrm{CH}-\mathrm{CH}_{2}}\left(t_{m} ; 0\right){\widetilde{\boldsymbol{H}_{\mathrm{d}}}}^{\mathrm{CH}-\mathrm{CH}_{2}}\left(t_{m} ; 0\right)\right] \rho^{(i)}\left(t_{1}\right),
\end{aligned}
$$

where $\tilde{\overline{\boldsymbol{H}}}_{\mathrm{d}}^{\mathrm{CH}-\mathrm{CH}_{2}}\left(t_{m} ; 0\right) \equiv \int_{0}^{t_{m}} \tilde{\overline{\boldsymbol{H}}}_{\mathrm{d}}^{\mathrm{CH}-\mathrm{CH}_{2}}(t) \mathrm{d} t$. The above density operator describes exactly the influence of the dipolar coupling of the methylene proton on the magnetization-exchange dynamics.

Taking into account the density operators that describe the spin system after application of the chemical-shift filters, Eqs. (7), (8) and Eq. (13), we can evaluate the normalized magnetization-exchange decays

and

$$
\left.\left\langle\left\langle I_{\mathrm{Z}} t_{m}\right)\right\rangle\right\rangle=1+\frac{1}{2}\left\langle\frac{\operatorname{Tr}\left\{\left[{\widetilde{\tilde{\boldsymbol{H}}_{\mathrm{d}}}}^{\mathrm{CH}-\mathrm{CH}_{2}}\left(t_{m} ; 0\right), I_{\mathrm{Z}}\right]^{2}\right\}}{\operatorname{Tr}\left\{I_{\mathrm{Z}}^{2}\right\}}\right\rangle
$$

$$
\left\langle\left\langle F_{\mathrm{Z}}\left(t_{m}\right)\right\rangle\right\rangle=1+\frac{1}{2}\left\langle\frac{\operatorname{Tr}\left\{\left[\tilde{\overline{\boldsymbol{H}}}_{\mathrm{d}}^{\mathrm{CH}-\mathrm{CH}_{2}}\left(t_{m} ; 0\right), F_{\mathrm{Z}}\right]^{2}\right\}}{\operatorname{Tr}\left\{F_{\mathrm{Z}}^{2}\right\}}\right\rangle .
$$

The initial behavior of the magnetization-exchange curves can now be explicitly evaluated from Eqs. (14) and (15). The initial decay rates of the diagonal signals are related to the formal second moment of the NMR absorption line of the respective functional group, where this absorption line is considered to be affected only by intergroup dipolar couplings and modulated by the chemical-shift and the residual dipolar coupling of the methylene protons. Using a straightforward operator algebra, we can finally write from Eqs. (14) and (15)

$$
\begin{aligned}
& \left\{\begin{array}{c}
\left\langle\left\langle I_{\mathrm{Z}}\left(t_{m}\right)\right\rangle\right\rangle \\
\left\langle\left\langle F_{\mathrm{Z}}\left(t_{m}\right)\right\rangle\right\rangle
\end{array}\right\} \\
& =1-\left\{\begin{array}{c}
1 \\
\frac{1}{2}
\end{array}\right\} \cdot\left\langle\left(\frac{\bar{D}^{\mathrm{CH}-\mathrm{CH}_{2}}}{3 \bar{D}^{\mathrm{CH}_{2}}-\delta}\right)^{2}\left\{1-\cos \left[\left(3 \bar{D}^{\mathrm{CH}_{2}}-\delta\right) t_{m}\right]\right\}\right\rangle .
\end{aligned}
$$

Here, $\delta \equiv \Delta \omega_{\mathrm{CH}}-\Delta \omega_{\mathrm{CH}_{2}}$ and $\bar{D}^{\mathrm{CH}-\mathrm{CH}_{2}} \equiv \bar{d}^{\mathrm{CH}-\mathrm{CH}_{2}} Y_{2,0}(\Omega)$. The cosine term in Eq. (16) shows the existence of so-called quantum-dynamical echoes [39-42]. These echoes originate at short mixing times from the coherent exchange of order between the Zeeman reservoirs and the dipolar reservoir (residual dipolar couplings) of the methylene protons. In the intermediate mixing-time regime they are superimposed on the quantum-dynamical echoes that are produced by the intergroup dipolar coupling. The statistical average finally leads to destructive interference of these echoes and, hence, to an apparently spin-diffusion-like decay. A common spin-diffusion process [2] takes place for long mixing times when the residual dipolar couplings along the chains are effective.

In the limit of even shorter mixing time, defined by $\left(3\left\langle\bar{D}^{\mathrm{CH}_{2}}\right\rangle-\delta\right) t_{m} \ll 1$, the trigonometric function can be expanded and the initial decays of the magnetization-exchange curves are simply

$$
\left\{\begin{array}{l}
\left.\left\langle\left\langle I_{\mathrm{Z}}\left(t_{m}\right)\right\rangle\right\rangle\right\rangle \\
\left\langle\left\langle F_{\mathrm{Z}}\left(t_{m}\right)\right\rangle\right\rangle
\end{array}\right\}=1-\left\{\begin{array}{c}
\frac{1}{2} \\
\frac{1}{4}
\end{array}\right\}\left\langle\left(\bar{D}^{\mathrm{CH}-\mathrm{CH}_{2}}\right)^{2}\right\rangle t_{m}^{2} .
$$


In this short mixing-time regime, the initial decay rates depend only on the intergroup residual dipolar coupling. We note that the longitudinal magnetization transfer from $\mathrm{CH}$ to $\mathrm{CH}_{2}$ is twice as fast as that from $\mathrm{CH}_{2}$ to $\mathrm{CH}$. This is related to the fact that the heat capacity of the $\mathrm{CH}_{2}$-Zeeman reservoir is twice as large as the heat capacity of the CH-Zeeman reservoir. Also, the size of the residual dipolar reservoir of the methylene protons is small compared with the Zeeman reservoirs.

\section{Experimental}

The investigated elastomer system is based on a cis-1,4-polybutadiene prepared and characterized at Max-Planck Institut für Polymerforschung in Mainz, Germany. The butadiene was polymerized by a conventional Ziegler-Natta polymerization at $40^{\circ} \mathrm{C}$ in a glass reactor with hexane as solvent. The uncross-linked sample was dissolved in toluene and subsequently dried. The microstructure was determined by ${ }^{13} \mathrm{C}-\mathrm{NMR}$ and showed a cis content of more than $97 \%$. Molecular weights were determined by GPC: $M_{n} \approx 130,000 \mathrm{~g} / \mathrm{mol}$ and $M_{w} / M_{n} \approx 4.5$. The glass transition temperature is $T_{\mathrm{g}} \approx 170 \mathrm{~K}$.

The NMR experiments were performed at a ${ }^{1} \mathrm{H}$ frequency of $300 \mathrm{MHz}$ on a Bruker MSL spectrometer using a variable temperature probe. The basic radio-frequency (rf) pulse sequence used in all the experiments is depicted in Fig. 3. The evolution period of duration $t_{1}$, the detection period $t_{2}$ as well as the mixing period $t_{m}$ and the dephasing delay $t_{\mathrm{d}}$ are shown. In combination with a CYCLOPS procedure, corrections for $T_{1}$ relaxation and multiple-quantum coherences are implemented in the phase cycle [2]. The initial magnetization-exchange rates (short mixing times) were measured using the longitudinal-magnetization-encoded dipolar echo [33]: The second half of the dipolar echo that appears at a time $t_{1}$ from the third pulse was recorded. The $\pi / 2$-pulse length of $3 \mu$ s and the recycle delay $2.5 \mathrm{~s}$ were used in all the experiments.

For the 1D magnetization-exchange experiments at very short mixing times, a static NMR probe was used that was adapted for microimaging with fast switching gradients (rise time smaller than $1 \mu \mathrm{s}$ ). A field gradient pulse with duration $t_{\mathrm{d}}=20 \mu$ s and strength $0.15 \mathrm{~T} \mathrm{~m}^{-1}$ was inserted after the second rf-pulse (cf. Fig. 3) to dephase the single- and multiple-quantum coherences that have been excited by the first two pulses.

In the 1D magnetization-exchange experiments, the duration $t_{1}$ for the chemical-shift filter was $260 \mu \mathrm{s}$ and the mixing time $t_{m}$ was incremented up to $200 \mathrm{~ms}$ for probing the exchange processes. For short mixing times $\left(t_{m}<10 \mathrm{~ms}\right)$, which will be analyzed in the following, the $T_{1}$ relaxation correction is not necessary.

\section{Results and discussion}

The 1D version of the magnetization-exchange experiment was performed in order to determine the residual dipolar couplings between the protons of the $\mathrm{CH}$ group and the $\mathrm{CH}_{2}$ group from the $t_{m}$ dependence of the peaks. Chemical-shift filters for the $\mathrm{CH}_{2}$ and $\mathrm{CH}$ lines have been implemented by appropriate choice of the rf-irradiation frequencies and $t_{1}$ (cf. Fig. 3). Figure 2 shows the two NMR spectra of the cis-1,4-polybutadiene sample that have been recorded with the 


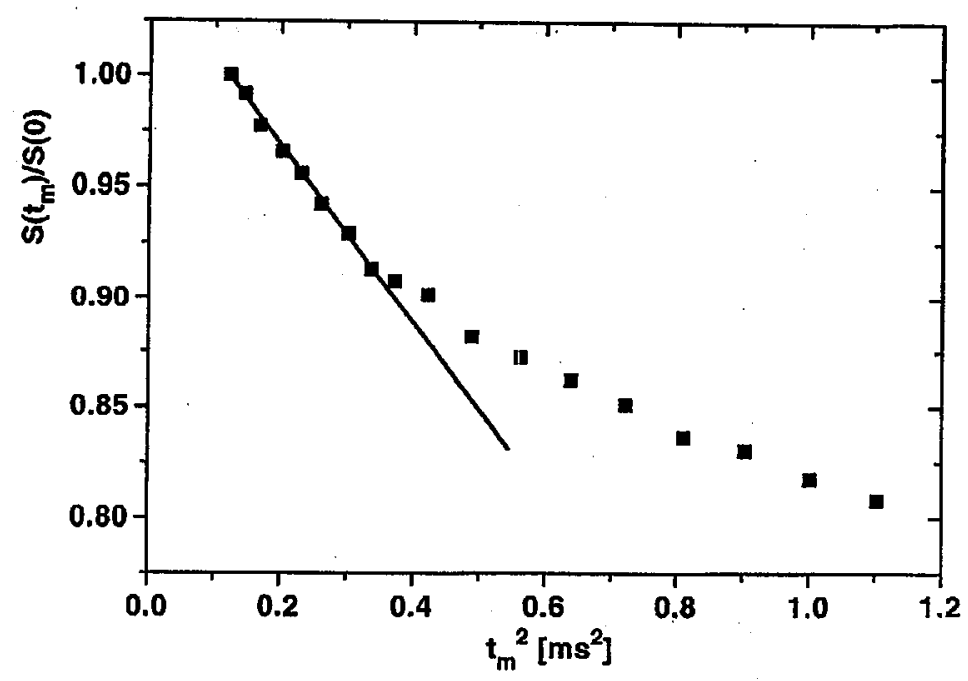

Fig. 4. The initial decays of the proton magnetization for the exchange process $\mathrm{CH} \rightarrow \mathrm{CH}_{2}$ using the chemical-shift filter for suppressing the $\mathrm{CH}_{2}$-lines and the pulse sequence of Fig. 3 with a dephasing gradient of duration $t_{\mathrm{d}}=20 \mu \mathrm{s}$. A static cis-1,4-polybutadiene sample was used. The initial part of the magnetization-exchange data (starting with $t_{m}=100 \mu \mathrm{s}$ ) shows the predicted linear dependence in $t_{m}^{2}$. The effective $\mathrm{CH}-\mathrm{CH}_{2}$ intergroup dipolar coupling constant $D_{\mathrm{eff}}^{\mathrm{CH}-\mathrm{CH}_{2}}$ can be evaluated by fitting of the theoretical curve Eq. (17).

three-pulse sequence for very short mixing time $\left(t_{m}=500 \mu \mathrm{s}\right)$ and for $t_{m}=15 \mathrm{~ms}$. The $\mathrm{CH}$ chemical-shift filter proved to be efficient in suppressing the $\mathrm{CH}_{2}$ line. For the long mixing time, the reappearance of the $\mathrm{CH}_{2}$ line is evident.

The decay of the longitudinal magnetization $M\left(t_{m}\right)$ normalized to the value $M_{0}$ that corresponds to zero mixing time, is recorded for short mixing times using the $\mathrm{CH}$ chemical-shift filter. According to Eq. (17), the magnetization-exchange dynamics should show an initial quadratic dependence on $t_{m}$. This dependence indeed is found in the experiments and is shown in Fig. 4. The solid-like behavior of the magnetization decay is valid for mixing times $t_{m} \ll\left(3\left\langle\bar{D}^{\mathrm{CH}_{2}}\right\rangle-\delta\right)^{-1}$. Using $\delta \approx 1 \mathrm{kHz}$ and $\left\langle\bar{D}^{\mathrm{CH}_{2}}\right\rangle \approx 1.2 \mathrm{kHz}$, as can be extracted from the line width at half-intensity of the proton $\mathrm{CH}_{2}$ line, we can estimate $\left(3\left\langle\bar{D}^{\mathrm{CH}_{2}}\right\rangle-\delta\right)^{-1} \approx$ $400 \mathrm{~ms}$. A deviation from the $t_{m}^{2}$ dependence is expected for mixing times $t_{m}>400 \mathrm{~ms}$, consistent with our data or: SBR discussed in Ref. [33].

In order to estimate the importance of the magnetization-exchange by cross-relaxation (NOE) processes for short mixing times, an analyze of the corresponding magnetization decay rates was done [33]. It was proved [33] that the initial magnetization-exchange process is dominated by residual dipolar couplings and not by the fluctuations of the dipolar interactions in the slow or fast motional regimes. 


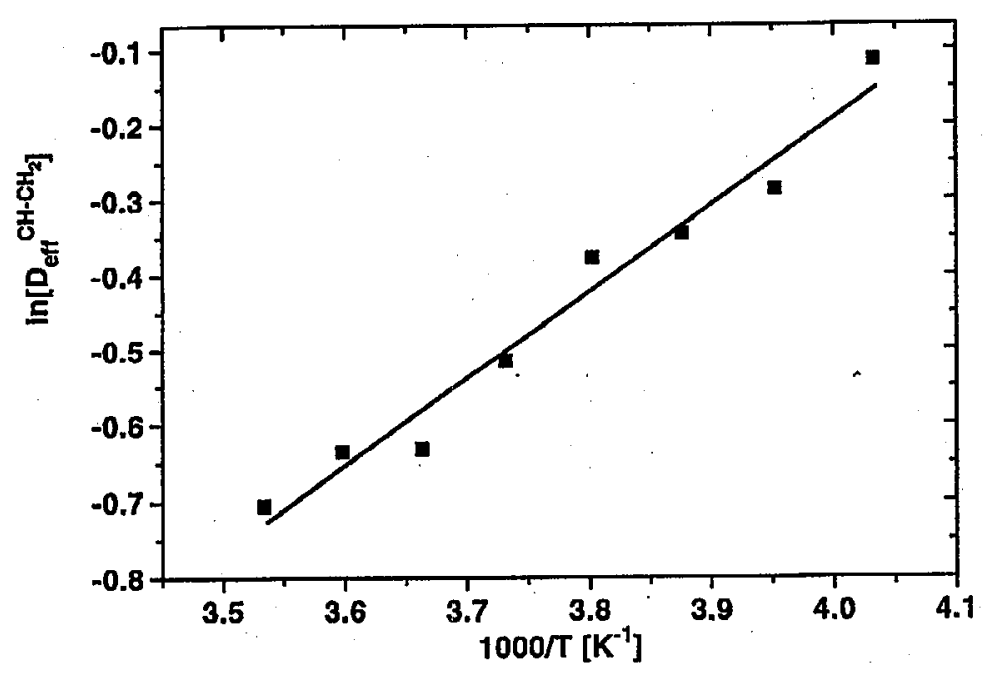

Fig. 5. The $\ln \left[D_{\mathrm{eff}}^{\mathrm{CH}-\mathrm{CH}_{2}}\right]$ versus inverse temperature for cis-1,4-polybutadiene elastomer.

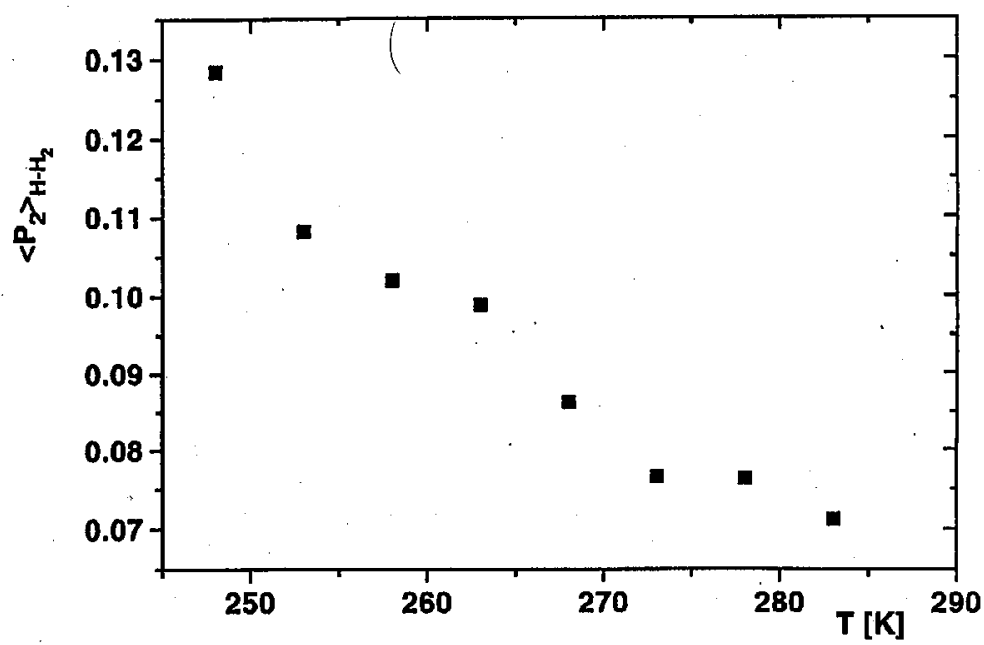

Fig. 6. The dynamic order parameter $\left\langle P_{2}\right\rangle_{\mathrm{H}-\mathrm{H}_{2}}$ evaluated using Eq. (18) and the data for residual dipolar coupling of Fig. 5, for cis-1,4-polybutadiene.

As described above, the values of the effective residual dipolar couplings $D_{\text {eff }}^{\mathrm{CH}-\mathrm{CH}_{2}}$ can be evaluated from the initial magnetization decays in the $1 \mathrm{D}$ exchange experiments. The measurements of $D_{\text {eff }}^{\mathrm{CH}-\mathrm{CH}_{2}}$ versus the temperature is presented in Fig. 5. In the investigated temperature range $245-285 \mathrm{~K}$, the temperature dependence can be in a good approximation fitted with an exponential function (cf. Fig. 5). In Sec. 3 it was discussed that the effective residual dipolar 
coupling scales with $N_{\mathrm{e}}^{-1}$. The correlation of the coupling constant $D_{\mathrm{eff}}^{\mathrm{CH}-\mathrm{CH}_{2}}$ with the shear modulus $G \propto N_{\mathrm{e}}^{-1}$, as an independent measure of the cross-link density demonstrates [33] this behavior. The temperature dependence of the intergroup dipolar residual coupling can be qualitatively explained by the fact that the physical cross-link density is connected with the temperature variation. From Eq. (3) it is evident that the number of the repeat units between the physical cross-link will increase as the temperature increases.

The dynamic order parameter can be evaluated from the residual dipolar

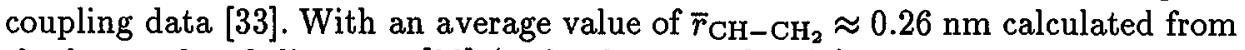
the known bond distances [33] (and references therein) $r_{\mathrm{CC}}=0.153 \mathrm{~nm}, r_{\mathrm{CH}}=$ $0.11 \mathrm{~nm}$ and $R_{\mathrm{HH}}=0.18 \mathrm{~nm}$ and bond angles $\alpha=119^{\circ}, \beta=109.5^{\circ}$ (cf. Fig. 1b) one can extract the value for the dynamic order parameter, $\left\langle P_{2}\right\rangle_{\mathrm{CH}}-\mathrm{CH}_{2}$, of the $\mathrm{CH}-\mathrm{CH}_{2}$ intergroup linkage $r_{\mathrm{CH}-\mathrm{CH}_{2}}$ according to

$$
\bar{D}_{\text {eff }}^{\mathrm{CH}-\mathrm{CH}_{2}}=\bar{D}_{\text {rigid }}^{\mathrm{CH}-\mathrm{CH}_{2}}\left\langle P_{2}\right\rangle_{\mathrm{H}-\mathrm{H}_{2}} \text {. }
$$

The dynamic order parameters evaluated from the above equation and the experimental data versus temperature are represented in Fig. 6.

\section{Conclusions}

The possibility to perform homonuclear separated-local field NMR spectroscopy by magnetization-exchange is discussed theoretically for a particular spin topology and proved experimentally.

1D magnetization-exchange spectroscopy was used for characterizing residual dipolar couplings in chemically uncross-linked $c i s$-1,4-polybutadiene. The residual dipolar coupling between the $\mathrm{CH}$ - and the $\mathrm{CH}_{2}$-group of butadiene was determined from the initial magnetization decay in a 1D exchange experiment. A simple model of three dipolar-coupled protons, which are arranged according to the $\mathrm{CH}-\mathrm{CH}_{2}$ chain topology, was used for describing the magnetization-exchange dynamics at small mixing times. The intergroup dipolar coupling is remarkably high in the uncross-linked elastomer and scales with the temperature. The quantitative understanding of this dependence is currently underway.

Magnetization-exchange NMR spectroscopy offers a convenient means for measuring the residual dipolar order between functional groups along the polymer chains. The use of protons, for which the principal axes of the dipolar tensor can be easily established, represents an important advantage of this method. It can be correlated with the local segmental order, which can also be determined by alternative NMR methods $[43,44]$. The technique proposed in this work uses spin polarization which evolves independently of dephasing effects arising from magnetic inhomogeneities or chemical shift. Dipolar interactions between selected spins can be measured as a result of the chemical selectivity offered by the static proton-NMR spectrum.

Magnetization-exchange experiments in the initial mixing time regime do not directly provide the residual dipolar couplings of the methylene protons or olefinic protons among themselves. These residual dipolar couplings, however, are accessible with high selectivity by applying the newly developed techniques of high-resolution multiple-quantum NMR spectroscopy performed under the conditions of fast MAS [28]. 
For the effect of the temperature and the correlation of the viscoelastic properties with NMR parameters of elastomers, the 1D version of the magnetization-exchange experiment has some advantages compared with alternative methods based on ${ }^{1} \mathrm{H}$ transverse relation, ${ }^{13} \mathrm{C}$-edited ${ }^{1} \mathrm{H}$ transverse relaxation and ${ }^{13} \mathrm{C}-{ }^{1} \mathrm{H}$ cross-polarization dynamics [31, 32, 44]. 1D magnetization-exchange experiments for protons are easy to implement and to analyze. The adverse effect of the chemical shift and the low efficiency of the cross-polarization are avoided. Moreover, magnetization-exchange experiments provide evidence that directly justifies the assumption of residual dipolar interactions which are localized to functional groups.

2D-exchange NMR spectroscopy in combination with MAS or homonuclear dipolar decoupling by a magic-sandwich sequence can separate the solid-like and liquid-like behavior of the polymer network and reveals the effects due to segmental motions.

Last, but not least, the magnetization-exchange decays can be used as a contrast parameter for molecular motion in NMR imaging experiments with elastomers. The residual dipolar couplings measured in a 1D exchange-experiment thus can provide a NMR parameter map for recording the spatial distribution of the cross-link density in rubber materials.

\section{Acknowledgments}

The authors acknowledge financial support from Volkswagen-Stiftung. The authors thank Prof. Dr. Hans W. Spiess and Dr. Siegfried Hafner for helpful comments.

\section{References}

[1] R.R. Ernst, G. Bodenhausen, A. Wokaun, Principles of Nuclear Magnetic Resonance in One and Two Dimensions, Clarendon Press, Oxford 1987.

[2] K. Schmidt-Rohr, H.W. Spiess, Multidimensional Solid-State NMR and Polymers, Academic Press, London 1994.

[3] J.S. Waugh, Proc. Natl. Acad. Sci. (USA) 73, 1394 (1976).

[4] R.K. Hester, J.L. Ackerman, B.L. Neff, J.S. Waugh, Phys. Rev. Lett. 36, 1081 (1976).

[5] E.F. Rybaczewski, B.L. Neff, J.S. Waugh, J.S. Sherfinski, J. Chem. Phys. 67, 1231 (1977).

[6] M.E. Stoll, A.J. Vega, R.W. Vaughan, J. Chem. Phys. 65, 4093 (1976).

[7] M. Alla, E. Lippmaa, Chem. Phys. Lett. 37, 260 (1976).

[8] S.J. Opella, J.S. Waugh, J. Chem. Phys. 65, 4919 (1977).

[9] M.E. Stoll, A.J. Vega, R.W. Vaughan, J. Chem. Phys. 65, 5458 (1978).

[10] M. Linder, A. Höhener, R.R. Ernst, J. Chem. Phys. 73, 4959 (1980).

[11] J. Schaefer, E.O. Stejskal, R.A. Mckay, W.T.J. Dixon, J. Magn. Reson. 52, 123 (1983).

[12] T. Terao, H. Miura, A. Saika, J. Chem. Phys. 85, 3816 (1986).

[13] W. Guo, B.M. Fung, J. Chem. Phys. 95, 3917 (1991).

[14] T. Nakai, T. Terao, Magn. Reson. Chem. 30, 42 (1992). 
[15] P. Palmas, P. Tekely, D. Canet, J. Magn. Reson. A 104, 26 (1993).

[16] K. Schmidt-Rohr, M. Wilhelm, A. Johansson, H.W. Spiess, Magn. Reson. Chem. 31, 352 (1993).

[17] K. Schmidt-Rohr, D. Nantz, L. Emsley, A. Pines, J. Phys. Chem. 98, 6668 (1994).

[18] M. Hong, K. Schmidt-Rohr, A. Pines, J. Am. Chem. Soc. 117, 3310 (1995).

[19] C.H. Wu, A. Ramamoorthy, S.J. Opella, J. Magn. Reson. A 109, 270 (1994).

[20] A. Ramamoorthy, S.J. Opella, Solid State NMR 4, 387 (1995).

[21] R. Tycho, G. Dabbach, Chem. Phys. Lett. 173, 461 (1990).

[22] D.P. Weitekamp, Adv. Magn. Reson. 11, 111 (1983) and references therein.

[23] S. Sinton, A. Pines, Chem. Phys. Lett. 76, 263 (1980).

[24] M. Gochin, K.V. Schenker, H. Zimmerman, A. Pines, J. Am. Chem. Soc. 108, 6813 (1986).

[25] D. Sandström, M.H. Levitt, J. Am. Chem. Soc. 118, 6966 (1996).

[26] W. Sommer, J. Gottwald, D.E. Demco, H.W. Spiess, J. Magn. Reson. A 112, 131 (1995).

[27] J. Gottwald, D.E. Demco, R. Graf, H.W. Spiess, Chem. Phys. Lett. 243, 314 (1995).

[28] D.E. Demco, J. Gottwald, R. Graf, M. Feike, S. Hafner, H.W. Spiess, Book of Abstracts, 37th ENC, Asilomar, 1996.

[29] M. Feike, D.E. Demco, R. Graf, J. Gottwald, S. Hafner, H.W. Spiess, J. Magn. Reson. A 122, 213 (1996) and references therein.

[30] S.A. Heffner, P. Mirau, Macromolecules 27, 7283 (1994).

[31] C. Fülber, D.E. Demco, O. Weintraub, B. Blümich, Macromol. Chem. Phys. 197, 581 (1996).

[32] P. Sotta, C. Fülber, D.E. Demco, B. Blümich, H.W. Spiess, Macromolecules 29, $6222(1996)$.

[33] D.E. Demco, S. Hafner, C. Fülber, R. Graf, H.W. Spiess, J. Chem. Phys. 105, 11285 (1996).

[34] J.A. Brydson, Rubber Chemistry, Applied Science Pub. Ltd, London 1978.

[35] J. Collignon, H. Sillescu, H.W. Spiess, Colloid Polym. Sci. 259, 220 (1981).

[36] P. Scotta, B. Deloche, Macromolecules 23, 1999 (1990).

[37] G.I. Sandakov, L.P. Smirnov, A.I. Sosikov, K.T. Summanen, N.N. Volkova, J. Polym. Sci. B, Polym. Phys. 32, 1585 (1994).

[38] A.D. English, Macromolecules 18, 178 (1985).

[39] F.M. McArthur, E.L. Hahn, R.E. Walstedt, Phys. Rev. 188, 609 (1969).

[40] D.E. Demco, J. Tegenfeld, J.S. Waugh, Phys. Rev. B 11, 4133 (1975).

[41] L. Müller, A. Kumar, T. Baumann, R.R. Ernst, Phys. Rev. Lett. 32, 1402 (1974).

[42] H.M. Pastawski, P.R. Levstein, G. Usaj, Phys. Rev. Lett. 75, 4310 (1995).

[43] H.W. Spiess, in: Structure and Properties of Oriented Polymers, Ed. I.M. Ward, in press.

[44] C. Fülber, D.E. Demco, B. Blümich, Solid State Nucl. Magn. Reson. 6, 213 (1996). 